\title{
L'ADOPTION DE LA PENSÉE SYSTÉMIQUE DANS LA GESTION PUBLIQUE : UN CHANGEMENT DE PARADIGME
}

\section{ARTICLE D'EXAMEN}

REBELO, Michele Alves Correa ${ }^{1}$

REBELO, Michele Alves Correa. L'adoption de la pensée systémique dans la gestion publique : un changement de paradigme. Revista Científica Multidisciplinar Núcleo do Conhecimento. 04 année, Ed. 10, vol. 01, pp. 45-63. octobre 2019. ISSN: 2448-0959, Lien d'accès: https://www.nucleodoconhecimento.com.br/administrationdes-affaires/pensee-systemique

\section{RÉSUMÉ}

Compte tenu du scénario politique brésilien actuel et des changements dans le panorama de la gestion publique au 21ème siècle, cet article vise à présenter les réflexions de l'adoption de la pensée systémique par le gestionnaire public qui démontre l'influence que cette pensée de décision. Elle est déduite d'une recherche de nature appliquée, d'un objectif exploratoire, avec l'adoption de la recherche bibliographique. II s'agit d'une question importante, en raison de la difficulté du gestionnaire public à atteindre l'efficacité dans l'exécution des activités administratives. Avec la recherche, il a été possible d'observer que la pensée systémique du gestionnaire influence l'orientation de la gestion publique, les avantages de cette posture peuvent être visibles dans les services fournis à la société et les actions internes de l'institution. Aujourd'hui, on observe la personnalité dans la prise de décision, en raison de l'influence politique de l'État et parce que c'est un scénario très compétitif, où la vanité personnelle et la culture de l'immédiateté prévalent. La pensée

\footnotetext{
${ }^{1}$ Maîtrise en gestion des politiques publiques - UNIVALI. Spécialiste en droit pénal et procédural-École de la magistrature de Santa Catarina. Spécialiste en gestion de la sécurité publique - UNIVALI. Diplôme de droit - FEBE.
} 
systémique contribue à ce que les nouvelles visions émergentes deviennent partagées et collaboratives. La gestion publique manque d'innovation systématique de la pensée pour répondre aux besoins sociaux et atteindre l'efficacité.

Mots-clés : gestion publique, gestionnaire public, principe d'efficacité, pensée systémique.

\section{INTRODUCTION}

La présente recherche propose de démontrer aux lecteurs les réflexions de l'adoption de la pensée systémique par le gestionnaire public et comment cela peut influencer la vie des gens.

Depuis la décennie 80 , un mouvement croissant a été observé à la recherche de techniques de gestion modernes et efficaces, capables d'améliorer les services publics (KETTL, 2005; PAULA, 2005; MANNING et col., 2009). Parallèlement à cette réalité, le gestionnaire public a rencontré un nouvel environnement mondial, de nouvelles exigences sociales, de nouvelles technologies et des concepts de projets.

En raison de ces facteurs, la façon d'administrer les services publics s'est transformée. Le modèle bureaucratique est remplacé par la gestion et les changements politiques, sociaux et culturels commencent à s'immiscer dans la façon de penser la société.

Malgré cette avancée dans les concepts de gestion et d'administration publique, il est perçu comme la difficulté du gestionnaire public à atteindre l'efficacité dans l'exécution de ses activités. Cela est dû à la priorisation des intérêts particuliers des candidats aux postes d'orientation, de leadership et de conseil, ainsi qu'à l'influence politique sur la prise de décisions des gestionnaires publics.

Selon le conseil (2009), la fonction d'orientation, rendue possible par l'accès à la position publique classée comme étant de la gratuité, implique des attentes quant à l'exercice de la position associée aux intérêts politiques de ceux qui ont le pouvoir de 
nommer. Pour cette raison, la loyauté personnelle est requise des personnes nommées. Il s'agit d'un lien précaire et peut être disculpé à tout moment.

Dans la tradition administrative brésilienne, seul le noyau le plus technique de l'État a tendance à avoir des postes de haute direction préservés des nominations nettement politiques (FLEURY, 2009). Idéalement, ces postes seraient occupés par des personnes qui possédaient des connaissances techniques. Cependant, il est adopté dans la plupart des cas, critère subjectif de nommage.

L'objectif de ce travail est de chercher une réflexion sur une nouvelle posture du gestionnaire public face à ce problème. II sera nécessaire d'aborder de façon superficielle des aspects de nature subjective, comme le caractère du gestionnaire, qui détermine le comportement moral ou social et, par conséquent, l'orientation d'une bonne ou d'une mauvaise gestion.

La gestion publique manque de nouvelles postures de pouvoir et de leadership. Une innovation systémique de la pensée devrait se produire à tout moment dans l'institution. II ne s'agit pas seulement de faire des choses différentes, mais de faire les mêmes choses de différentes manières, en créant, comme le mentionne Peter Drucker (2002), de nouveaux potentiels de satisfaction.

Compte tenu de la complexité du thème et d'une analyse systématique, nous avons le droit et le gestionnaire en tant que systèmes ouverts, qui se rapportent dans un accord age dynamique. Ils sont dans un processus continu et incessant d'échanges et d'échanges avec l'environnement. Les organisations sont des systèmes de rôles joués par les gens (CHIAVENATO, 2000).

Sous la vision et la contribution des sciences sociales, il y a la possibilité de mener la recherche basée sur la théorie de Bertalanffy (1937), qui a une vision différente du réductionnisme scientifique jusqu'alors appliqué par la science conventionnelle. 
Selon Valença (2011), l'approche systémique est directement associée à la lecture de la complexité du raisonnement, de la maîtrise ou de la supervenience de l'émotion dans les décisions humaines et les stimuli à la décision efficace.

Penser systématiquement, c'est penser à la complexité, à l'instabilité et à l'intersubjectivité. Un professionnel qui vit - voit le monde et agit en elle - les implications d'avoir assumé les hypothèses susmentionnées, peut être considéré comme un professionnel systémique. II doit avoir le regard de l'observateur l'Observateur dans le cadre du système (VASCONCELOS, 2009).

Avec le thème décrit, il est proposé de démontrer les réflexions de l'adoption de la pensée systémique et les influences que cette posture peut générer dans la vie des gens, répondant à la question suivante: il sera possible d'atteindre l'efficacité avec l'adoption de la pensée systémique par Gestionnaire public ?

Pour atteindre l'objectif proposé, la méthode descriptive sera utilisée par la recherche bibliographique.

Pour Fonseca (2002), la recherche bibliographique est faite à partir de l'enquête sur les références théoriques déjà analysées et publiées par des moyens écrits et électroniques, tels que des livres, des articles scientifiques et des pages de sites Web.

Dans le chapitre suivant sera présenté le concept de gestion publique et, en question, le gestionnaire. Le principe d'efficacité sera alors abordé de manière superficielle. Enfin, nous présenterons la réflexion systémique sur ce regard dans la construction d'un nouveau paradigme de gestion publique.

\section{GESTION PUBLIQUE}

Au cours des 30 dernières années, le terme « gestion publique » a été utilisé pour remplacer l'administration publique et, pour cette raison, a été considéré comme un terme polysémique, puisqu'une partie de la doctrine comprend que les deux termes sont erronés. D'autres soutiennent qu'il y a eu une perturbation du concept traditionnel, 
parce qu'il englobe les outils de gestion du monde des affaires et, par conséquent, un concept plus large.

Henri Fayol (1950), déjà dans la décennie des années 50, définissait la gestion comme des fonctions administratives de prévision, d'organisation, de commandement, de coordination et de contrôle.

Depuis la décennie 80, les gouvernements de plusieurs pays ont entrepris des efforts pour moderniser l'administration publique afin de réduire la portée du gouvernement et de le faire fonctionner mieux dans la poursuite de l'agilité. L'évaluation de l'administration publique elle-même change, puis est balisée par des critères proches de ceux utilisés par l'administration privée.

À la suite de cette posture se pose le Nouveau Manegement Public (NPM), associé au contexte d'une réforme spécifique de l'Etat, dont le modèle est opposé à l'administration publique conventionnelle, basée sur le modèle de bureaucratie weberianeinjured consiste à conférer une Approche managériale, inspirée par les méthodes de gestion des entreprises privées, à l'administration publique (HOOD, 1995; HERNES, 2005), afin d'assurer une plus grande réactivité et une meilleure performance dans la prestation de services publics à la population (MANNING et al., 2009).

Perry et Kraemer (1983) Considérer que la gestion publique est une fusion de l'orientation normative de l'administration publique traditionnelle et de l'orientation instrumentale de la direction, dans un sens générique. En d'autres termes, dans le premier aspect, la gestion publique intègre des thèmes tels que la démocratie et la responsabilité, et des valeurs telles que l'équité, l'égalité et la probité. Et dans le second, une orientation instrumentale, avec l'idée que le secteur public partage avec le privé la nécessité d'atteindre ses objectifs d'une manière plus économique et efficace.

Pour Druker (1993), la direction ne fait pas référence à la hiérarchie organisationnelle d'une administration classique, mais à la capacité de promouvoir l'innovation 
systématique des connaissances et d'en tirer le rendement maximal dans son application à la production.

Contrairement à la gestion privée, la gestion publique doit être guidée par des valeurs sociales. On suppose ici, la conception que la gestion est un acte complexe qui nous rapproche du monde de la politique (BRUGUÉ; SUBIRATS, 1996).

Penser à l'évolution historique de la gestion publique, prospectivement, se réfère à une réflexion sur le véritable rôle de l'Etat de nos jours. La gestion de ce qui est public est un acte complexe et difficile parce que le scénario est très concurrentiel. La vanité personnelle et la culture de l'immédiateté prévalent dans ce milieu. Chaque législature, la dynamique de l'administration est modifiée. II n'y a pas de continuité des actions en raison de la personnalité dans la prise de décision, compte tenu de l'influence politique qui délire le pays.

Les gens sont terrifiés par les nouvelles de crimes barbares qui sont conveed dans les médias. Cependant, ils ne se rendent pas compte qu'un travail hors de prix ou inachevé, en raison d'une mauvaise gestion, peut avoir contribué au chaos dans l'éducation et la santé d'une région particulière.

Cardoso Jr (2001) soutient que la responsabilité de l'État n'est pas seulement de faire les choses mieux et plus efficacement. Plus que cela, il s'agit d'induire, de favoriser ou même de produire les conditions propices à la transformation des structures économiques et sociales du pays. Le processus comprend la refonte de la structure, des procédures et des pratiques du secteur public, en intégrant des changements de grande ampleur dans la dimension institutionnelle, qui comprennent des questions liées aux principes et aux valeurs qui guident la relation de ces organisations. Avec la société et le marché (FERLIE et coll., 1999; Ormond LOFFLER, 1999; HERNES, 2005).

En ce sens, la gestion publique manque d'innovation systémique pour répondre aux besoins sociaux et atteindre l'efficacité. L'adoption de la pensée systémique par le gestionnaire peut contribuer à faire en venir à de nouvelles visions émergentes et à 
devenir des visions partagées et collaboratives. La vision de l'ensemble est fondamentale pour la prise de décision.

Pour Abrôcio (2007), la gestion publique a un certain nombre de particularités qui se rapportent à la nécessité d'avoir des instruments de gestion et démocratiques pour lutter contre les problèmes auxquels l'État est confronté dans le monde contemporain. Dans ce contexte, le formalisme et la rigidité bureaucratique devraient être attaqués comme des maux, et la méritocratie peut promouvoir la modernisation.

La vision partagée est la première étape pour amener les gens qui ne se font pas confiance à commencer à travailler ensemble. Crée une identité commune. (SENGE,2009). Grâce à une culture de rétroaction, la relation de confiance dans l'équipe et la reconnaissance de l'effort contribuent à l'efficacité de la gestion publique, car elle atteint l'aspect lié à la motivation.

Le gestionnaire peut transformer les concepts (pré) établis tout au long de l'histoire. En dépit d'être lié au principe de légalité, vous pouvez faire une différence. Pour cela, il doit assumer une nouvelle posture, le regard systémique dans la poursuite de l'efficacité, dont les concepts et les aspects seront discutés ci-dessous.

\subsection{GESTIONNAIRE PUBLIC}

Selon le concept classique développé par Henri Fayol (1950), le gestionnaire est défini par ses fonctions au cours de l'organisation. C'est la personne qui est responsable de l'interprétation des objectifs proposés par l'institution et qui agit par la planification afin d'atteindre les buts et les objectifs.

On peut alors dire que le gestionnaire est quelqu'un qui élabore le plan stratégique et opérationnel, juge les moyens efficaces, conçoit les structures et établit les règles et procédures politiques les plus appropriées et, en fin de compte, met en œuvre et coordonne la mise en œuvre des projets par Le milieu d'un type particulier de commandement (ou de leadership). 
Pour Chiavenato (2004), être gestionnaire ou leader, c'est avoir une vision globale, une relation entre l'homme et son environnement de travail. En outre, c'est apprendre à enseigner et à apprendre, étant ce dernier d'une importance vitale. Partant d'une prémisse plus subjective, Peter Drucker (1993) affirme que c'est par le caractère qui est exercé le leadership. Dans la même ligne de pensée, Havard (2011) définit que la vertu, en plus d'être une valeur intrinsèque de l'être humain, est une force dynamique qui augmente la capacité d'action, une caractéristique qui est si nécessaire au leader. La vertu crée la confiance, et sans confiance, il devient impossible de diriger.

Le fait d'être gestionnaire public est un défi, parce que la gestion de quelque chose qui est public est un acte complexe en raison du paysage politique hautement concurrentiel. Compte tenu des gros titres fréquents convélaient dans les médias de la corruption, des écarts par rapport aux fonds publics, un paradigme a été établi que les services publics ne servent pas la société efficacement.

À chaque législature, la dynamique de l'administration est modifiée, avec des changements radicaux qui se produisent. Ce processus est salutaire, parce que sinon il y aurait la dictature ou la monarchie. Cependant, une continuité des actions et des projets est nécessaire. Il est très important que le gestionnaire ait la science et la notion de l'importance de changer de posture dans l'exécution des tâches quotidiennes et comment cette attitude peut transformer la vie des gens. Une sage décision peut signifier l'éducation des enfants dans le besoin dans une région particulière. Plus que jamais, la société réclame des résultats différents. II est nécessaire d'examiner le processus traditionnel de réflexion de la fonction publique et de mise en œuvre de nouvelles attitudes.

Le gestionnaire public de la même manière que dans l'initiative privée a besoin des caractéristiques et des qualités qui lui permettent de promouvoir les changements qui sont proposés. En tant que chefs de file du secteur public, les gestionnaires doivent être dotés de connaissances, de compétences et d'attitudes, ainsi que d'une forte vocation à s'occuper des diverses formes et sources de pouvoir qui imprègnent le secteur public (MORGAN, 1996). 
L'adoption de la pensée systémique est intimement liée au développement des vertus éthiques du gestionnaire et/ou du leader. Nous pouvons relier certaines caractéristiques aux aspects systémiques, à savoir : le leadership axé sur la coopération et l'influence, la communicabilité en mettant l'accent sur les relations, l'humanitanisme en mettant l'accent sur le processus, la pensée de réseau, la flexibilité, Vision large avec totalité et dynamisme (ANDRADE, 2016).

L'action de l'administration est liée à la pratique de la pensée systémique et de l'effet de levier stratégique. Les caractéristiques du leadership, dans le contexte de l'interaction humaine qui favorise les processus d'optimisation de l'apprentissage, sont orientées vers l'exercice de la participation à tous les niveaux et vers la construction d'équipes (ZANELLI,2008).

L'idée de l'inefficacité des services publics devrait être abandonnée et l'influence politique réduite. II est nécessaire de comprendre que si l'attention est focalisée sur une partie isolée visant des intérêts restreints, il ne sera pas possible de percevoir l'ensemble du système, étant fondamental de penser à l'ensemble et non dans les phases, si vous voulez corriger les orientations et atteindre l'efficacité, dont le thème sera affiché dans le sujet suivant.

\section{PRINCIPE D'EFFICACITÉ}

Au départ, il est nécessaire d'établir que les principes administratifs sont des lignes directrices de base qui corroborent et appuient la façon de faire fonctionner les agents publics, lorsque les attributions liées à l'administration publique sont efficaces.

Le principe d'efficacité, prévu dans l'art. 37 des FC de 1988, ont eu leur origine dans le droit privé, étant insérédans dans l'administration publique, dans la réforme administrative.

L'efficacité dans le secteur public est une exigence de la nouvelle tendance mondiale pour répondre rapidement aux intérêts collectifs et avec des résultats efficaces. II cherche un État qui planifie, développe et exécute efficacement ses fonctions, en 
faisant usage de nouvelles techniques et habitudes qui visent des résultats satisfaisants.

Le principe d'efficacité peut être compris en deux biais : d'abord par rapport au mode d'action de l'agent public, qui est attendu comme la meilleure exécution possible de ses attributions, visant les meilleurs résultats et, d'autre part, par rapport à la manière d'organiser, Structurer et discipliner l'administration publique, également dans le but d'obtenir les meilleurs résultats dans la prestation de la fonction publique (DI PIETRO, 2014).

L'activité administrative doit être menée par des gestionnaires engagés et soucieux de l'efficacité de la prestation des services publics. La principale caractéristique du gestionnaire devrait être la pensée systémique, comme un moyen de visualiser l'ensemble du processus. Une gestion efficace a le développement social comme point de forme. Par conséquent, l'objectif principal du gestionnaire devrait être lié à l'utilisation des meilleurs moyens de satisfaire les besoins collectifs.

Le changement de posture est lié à la nouvelle vision des gestionnaires et des citoyens par rapport à l'administration de la chose publique. Il est nécessaire de se détacher de l'opinion désuète selon laquelle l'intérêt de l'État chevauche l'intérêt privé.

Le constitutionnalisme moderne a non seulement placé l'homme à l'épicentre de l'ordre juridique, garantissant une protection différenciée des droits fondamentaux individuels, mais aussi des intérêts de nature collective, qui surpassent la sphère individuelle, L'objectif de permettre la jouissance des droits par tous les membres de la communauté politique (BINENBOJM,2010).

Le terme intérêt public doit être interprété comme la réalisation maximale de tous les intérêts, individuels et collectifs, protégés par la loi. Dans l'état de droit démocratique actuel, les droits fondamentaux de la société se chevauchent et leur satisfaction doit être poursuivie par l'État au-dessus de tout autre intérêt. 
Le manque de contrôle de la société sur l'État, encore dominé par des intérêts particuliers, est indiqué comme un facteur négatif dans la gestion publique. Pour le Fils (2005), la préservation de la sphère privée de l'individu, associée à la promotion des désirs de la communauté politique, représente l'intérêt public réel à poursuivre par l'administration.

Pour que l'efficacité s'améliore de manière significative, non seulement la démocratisation du système politique est nécessaire, mais aussi l'inclusion de mécanismes démocratiques internes à l'administration publique, qui sont liés à la formation de politiques publiques et à la Partage du pouvoir dans la prise de décision.

Les gestionnaires publics, les citoyens et les autres acteurs font partie d'un système de gouvernance dynamique, ouvert et interactif, où l'autorité de l'État est utilisée de différentes façons pour obtenir divers résultats publics.

Dans ce contexte, la participation de la société a une valeur incommensurable, car elle encourage la citoyenneté active. L'autonomisation des communautés et l'esprit civique peuvent faire une différence dans la gestion publique et, par conséquent, dans les résultats des services publics fournis par l'État.

D'autre part, le gestionnaire public doit refléter ses actions, sous l'égide de la pensée systémique, dont le thème sera discuté ci-dessous.

\section{PENSÉE SYSTÉMIQUE}

Par l'acte de penser, les êtres humains façonnent le monde, interagissant selon leurs désirs, leurs plans et leurs objectifs. Les concepts et les processus de la cognition, de la sensibilité, de la conscience et de l'imagination (ANDRADE, 2016) peuvent être associés à la pensée. En mettant l'accent sur l'évolution de la science, nous pouvons citer les pensées cartésiennes, complexes et systémiques prédominantes.

La pensée systémique a surgi au siècle twentieth, opposé à la pensée réducteurmécaniste de Descartes et Newton, qui est basée sur le paradigme scientifique 
traditionnel mentionné par Vasconcellos (2009), dont les hypothèses de base sont résumées dans Simplicité, stabilité et objectivité.

L'hypothèse de la simplicité repose sur la conviction qu'il est nécessaire de séparer les parties pour comprendre l'ensemble. Dans ce regard, Cunha (2006) interprète que la réduction est une autre opération en quête de simplicité, qui unifie ce qui est diversifié. En trouvant un phénomène complexe, le scientifique cherche sa réduction à un autre phénomène plus simple et déjà bien compris.

L'hypothèse de la stabilité réside dans la croyance qu'il y a de la stabilité dans le monde et qu'il y a régulièrement des répétitions. Dans l'hypothèse de l'objectivité, il constitue la conviction qu'il est possible de connaître le monde tel qu'il est composé en réalité, compte tenu du critère essentiel de la pensée scientifique (CUNHA, 2006).

Le paradigme de la science traditionnelle, à travers la pensée linéaire-cartésienne, s'est développé et s'est établi dans les sciences physiques et exactes, devenant un modèle de science. Les physiciens ont agi sur la base des hypothèses de simplicité, de stabilité et d'objectivité, et ont réussi, expliquant le monde physique et développant des technologies sophistiquées qui modifiaient de plus en plus les relations de I'Homme avec la nature (VASCONCELLOS, 2009).

Avec la conviction qu'il y a une différence entre les êtres humains et la nature, et que les phénomènes humains sont de nature subjective, la rupture entre les sciences de la nature (objectives) et humaines a été établie. Selon Vasconcellos (2009), les sciences physiques ont facilement adopté les trois hypothèses épistémologiques. Les sciences biologiques ont adopté l'hypothèse de l'objectivité, et ont eu des problèmes avec ceux de simplicité et de stabilité, tandis que les sciences humaines ont eu des difficultés par rapport aux trois hypothèses, en particulier, celle de l'objectivité.

La pensée systémique propose, en revanche, les paradigmes de complexité, d'instabilité et d'intersubjectivité, qui s'intègrent incroyablement à la psychologie analytique de Carl Gustav Jung (VASCONCELLOS, 2009). 
Un professionnel qui vit, voit le monde et y agit les implications d'avoir assumé pour lui-même les hypothèses de complexité, d'instabilité et d'intersubjectivité peut être considéré comme un professionnel systémique (VASCONCELLOS, 2009).

Le paradigme systémique, qui considère les hypothèses de complexité, de subjectivité, d'instabilité, a comme principes la flexibilité et la plasticité qui se manifestent dans les systèmes à travers les caractéristiques auto-organizativas qui, à partir du chaos, Suppose les aspects de l'auto-renouvellement et de l'autotranscedence. Ainsi, des systèmes ouverts loin de l'équilibre peuvent préserver, reproduire, renouveler, s'adapter et évoluer. Ainsi, les processus d'adaptation d'un côté et de l'autre sont évidents, ceux de l'apprentissage, du développement et de l'évolution (COSTA, 2007).

Cunha (2006) exprime l'idée que la pensée systémique signifie penser en termes de connexions, de relations, de contexte, d'interactions des éléments d'un tout; Pour voir les choses en termes de réseaux, de réseaux et de communautés.

La pensée systémique ne nie pas la rationalité scientifique, mais estime qu'elle ne fournit pas des paramètres suffisants pour le développement humain et pour la description de l'univers matériel. Pour cette raison, il cherche à regarder dans la direction opposée du réductionnisme, en accordant plus d'importance à l'ensemble qu'à l'autre.

II présuppose une méthodologie de processus de pensée, qui configurent l'existence de l'ensemble. C'est-à-dire qu'il est nécessaire d'étudier les systèmes à l'échelle mondiale, afin d'impliquer toutes leurs interdépendances, parce que chacun des éléments, lorsqu'ils sont assemblés pour constituer une unité fonctionnelle plus grande, développent des qualités qui ne sont pas dans leurs composantes Isolats (BERTALANFFY, 2009).

Capra (1996) soutient que la pensée fragmentée n'est pas capable de s'attaquer à l'interdépendance des problèmes mondiaux, tant au niveau supérieur de la société qu'au niveau individuel. En ce sens, l'essence de la pensée systémique est dans le

Disponível em: https://www.nucleodoconhecimento.com.br/administration-des-affaires/pensee- 
changement de mentalité, ce qui signifie voir les interrelations au lieu des chaînes linéaires de cause-effet et voir des processus de changement plutôt que des instantanés (Senge, 2009). Dans cette réunion, les changements ne doivent pas être considérés comme des actions isolées, mais comme un ensemble de mesures interdépendantes qui font partie de la planification des améliorations de l'organisation.

C'est une façon d'analyser et de penser les connaissances sur le monde, à travers les systèmes. Une vision systémique ou une "pensée systémique " signifie garder à l'esprit ce que l'on veut résoudre, choisir la bonne ou la manière la plus appropriée. On peut dire qu'il s'agit d'une compétence qu'une personne acquiert pour analyser les événements et leurs conséquences possibles, dans le but de créer une solution unique qui contemple les attentes de toutes les parties concernées.

Dans la gestion privée et publique, il n'y a pas d'uniformité dans l'adoption de la pensée systémique par les gestionnaires. La plupart des agents publics ne connaissent même pas le concept. On peut réaffirmer l'importance de la pensée systémique dans la gestion à travers les mots de Zanelli:

L'action de l'administration est liée à la pratique de la pensée systémique et de l'effet de levier stratégique. Les caractéristiques du leadership, dans le contexte de l'interaction humaine qui privilégie les processus d'optimisation de l'apprentissage, sont orientées vers l'exercice de la participation à tous les niveaux et vers la construction d'équipes (ZANELLI, 2008, p. 13).

En 2008, la National Quality Foundation (FNQ), présente dans les modèles d'excellence les plus prestigieux du monde, a mené une enquête virtuelle auprès de 196 cadres brésiliens. Les résultats ont révélé que la majorité des répondants ne connaissaient que partiellement le concept de pensée systémique.

La grande préoccupation des scientifiques systémiques est la reconnaissance du fait que la nécessité d'un changement profond de perception et de pensée, pour assurer la survie, n'a pas encore atteint la majorité des gestionnaires, ni les administrateurs et les enseignants de Nos grandes universités (CAPRA, 1996).

Disponível em: https://www.nucleodoconhecimento.com.br/administration-des-affaires/pensee- 
Lorsque les gens perçoivent le monde différemment, de nouvelles croyances et hypothèses émergent, ce qui permet le développement de nouvelles compétences et capacités, formant un cycle continu d'apprentissage organisationnel.

Peter Senge (2009) Place que la pensée systémique peut être présentée comme une discipline, une étude avec la théorie et la pratique, qui permet de développer la vision de l'assemblage, une structure pour voir les interrelations à la place des choses, pour voir les modèles de changement Au lieu de statique ' instantanés '. Le gestionnaire, en adoptent la pensée systémique, peut faire une différence dans la gestion publique et changer la réalité du pays. Avec ce nouveau look, vous pouvez mener les actions de l'institution, interpréter les politiques et les procédures et adapter les processus et les pratiques internes aux exigences définies par la loi.

II faut de l'innovation dans la gestion publique. Le gestionnaire doit assumer le rôle et cesse d'agir comme un simple soutien, l'exécution des tâches avec courage. Penser systématiquement au nom de l'aide sociale.

II s'agit d'un thème complexe, parce que le changement de posture du gestionnaire, en adoptant la pensée systémique dans l'exercice de la gestion, est lié à des critères subjectifs, tels que le caractère, dont la vertu n'est pas mesurée ou évaluée lors de l'entrée de cette Professionnel dans l'activité publique, indépendamment du fait qu'il s'agisse d'un appel d'offres public ou d'une nomination par nomination.

Toutefois, tel que présenté dans la recherche, la gestion publique progresse de plus en plus, ce qui accroît la capacité politique de l'État à « bien faire la politique » pour mieux servir la société et, par conséquent, atteindre l'efficacité. De plus en plus d'auteurs préconisent l'abandon théorique du principe de suprématie. Les intérêts publics et privés ne sont manifestement pas des catégories antagonistes, mais plutôt complémentaires (JUSTEN FILHO, 2005).

La recherche nous a permis d'inférer que l'application des concepts de pensée systémique peut ajouter une valeur significative à plusieurs domaines de recherche et dans les secteurs d'organisations. En ce sens, l'utilisation de l'approche systémique 
dans d'autres domaines peut être révélée comme une contribution académique pertinente.

Il est donc valable d'affirmer que l'objectif de cette étude a été atteint, et il est possible d'affirmer que l'adoption de la pensée systémique par le gestionnaire est directement liée au principe d'efficacité.

Pour les études futures, il est proposé de mener des recherches dans les organes de l'administration publique, dans le but de vérifier les caractéristiques comportementales systémiques des gestionnaires, en suggérant des pratiques à la gestion systémique en mettant l'accent sur le comportement des gens .

\section{CONSIDÉRATIONS FINALES}

Après avoir analysé les documents trouvés dans la littérature, il a été possible d'identifier que l'adoption de la pensée systémique par le gestionnaire peut influencer I'orientation de la gestion publique du pays. II s'agit d'une compétence qu'une personne acquiert pour analyser les événements et leurs conséquences possibles, dans le but de créer une solution unique qui contemple les attentes de toutes les parties concernées. Les avantages de cette nouvelle vision/posture peuvent être visibles dans les services rendus à la société et les actions internes de l'institution, car elle est directement liée à la prise de décision.

À cette fin, le gestionnaire public, de la même manière que dans l'initiative privée, a besoin des caractéristiques et des qualités qui lui permettent de promouvoir les changements qui sont proposés. En tant que dirigeants du secteur public, les gestionnaires doivent être dotés de connaissances, de compétences et d'attitudes, ainsi que d'une forte vocation à s'occuper des diverses formes et sources de pouvoir qui imprègnent le secteur public (MORGAN, 1996).

L'adoption de la pensée systémique est intimement liée au développement des vertus éthiques du gestionnaire. La vision du monde est individuelle. Bientôt, il n'y a aucun moyen d'avoir une unité ou un contrôle dans la façon de penser et d'agir. Dans cette

Disponível em: https://www.nucleodoconhecimento.com.br/administration-des-affaires/pensee- 
conjoncture, il ne faut pas penser que les changements sont des actions isolées, mais comme un ensemble de mesures indépendantes qui font partie de la planification des améliorations de l'organisation.

La pensée systémique " aide à comprendre comment changer les systèmes plus efficacement et comment agir en meilleure harmonie avec les processus du monde naturel et social » (VALENÇA, 2011).

Les organisations qui réussiront vraiment à l'avenir seront celles qui découvriront comment cultiver chez les gens l'engagement et la capacité d'apprendre à tous les niveaux de l'Organisation (SENGE,2009).

Par conséquent, il convient de considérer que la présente étude a l'intention de discuter d'une perspective encore naissante sur un thème stratégique pour les organismes publics en termes de gestion, sans intention d'épuiser le sujet.

\section{RÉFÉRENCES}

ABRUCIO, Fernando Luiz. Trajetória recente da gestão pública brasileira: um balanço crítico e a renovação da agenda de reformas. Edição Especial Comemorativa. Rio de Janeiro: Revista de Administração Pública (RAP), 2007.

ALVAREZ, Maria Esmeralda Ballestero. Organização, sistemas e métodos. São Paulo: McGraw-Hill, 1990.

ALVES, João Bosco da Mota. Teoria Geral de Sistemas - Em busca da interdisciplinaridade. Florianópolis. Instituto Stela, 2012.

AMARAL, Antônio Carlos Cintra do. O princípio da eficiência no direito administrativo. Revista Diálogo Jurídico, Salvador, CAJ - Centro de Atualização Jurídica, n.14, jun./ago. 2002. 
ANDRADE, A; Rodrigues, L; Seleme A; Souto, R; Pensamento sistêmico: Caderno de campo. $O$ desafio da mudança sustentada nas organizações e na sociedade. Porto Alegre: Bookman, 2006.

BINENBOJM, Gustavo. Da Supremacia do Interesse Público ao Dever de Proporcionalidade: Um novo paradigma para o Direito Administrativo. In: SARMENTO, Daniel (org.). Interesses públicos versus Interesses privados: desconstruindo o princípio da supremacia do interesse público. Rio de Janeiro: Lumen Juris, 2010.

BERTALANFFY, L. von. Teoria Geral dos Sistemas: Fundamentos, desenvolvimento e aplicações. São Paulo: Editora Fundação Perseu Abramo, 2006.

BRASIL. Constituição da republica Federativa do Brasil: 1988. 25. Ed. Brasília: Câmara dos Deputados, Coordenação de Publicações, 2012.

CÂMARA, Leonor Moreira. Fatores do Pensamento Sistêmico como Potencializadores de Sucesso de Projetos de Software no Setor Público.organização da administração pública Federal Brasileira: uma introdução ao estudo da organização da direção pública na perspectiva de estudos organizacionais. Rio de Janeiro: Revista de Administração Pública (RAP), 43(3), maio/junho 2009.

CAPRA, F. O Ponto de Mutação. A ciência, a sociedade e a cultura emergente. Trad. Álvaro Cabral, São Paulo: Cultrix, 2006.

CAPRA, Fritjof. A Teia da Vida. Uma Compreensão Cientifica dos Sistemas Vivos. São Paulo (SP). Editora Cultrix. 1997.

CARNEIRO, R., MENICUCCI, TMG. Gestão pública no século XXI: as reformas pendentes. In Fundação Oswaldo Cruz. A saúde no Brasil em 2030 - prospecção estratégica do sistema de saúde brasileiro: desenvolvimento, Estado e políticas de saúde [online]. Rio de Janeiro: Fiocruz/lpea/Ministério da Saúde/Secretaria de 
Assuntos Estratégicos da Presidência da República, 2013. Vol. 1. pp. 135-194. ISBN 978-85-8110-015-9. AvailablefromSciELO Books.

CHIAVENATO, Idalberto. Recursos Humanos. O Capital Humano das Oganizações. SãoPaulo:Atlas, 2004.

CHIAVENATO, Idalberto. Iniciação à Organizações e Controle.São Paulo: McGrawHill, 1989.

COSTA, WedjaJosefa Granja. Socionomia de base sistêmica: Método de apoio à gestão de grupos na organização. Dissertação de Mestrado. Fortaleza: Universidade Federal do Ceará, 2007.

CUNHA, Aura Celeste Santana. Pensamento Sistêmico e tecnologia educacional: a metodologia WEBQUEST. Dissertação de Mestrado Profissional em Computação. Fortaleza: Universidade Estadual do Ceará, 2006.

DRUCKER, Peter. The effective executive. Harper Collins Publishers, 1993.

DRUCKER, Peter F. Administrando para o Futuro: os anos 90 e a virada do século. Tradução de Nivaldo Montigelli Jr. São Paulo: Pioneira Thomson Learning, 2002.

FALCONI, Vicente. O verdadeiro Poder.Nova Lima: INDG Tecnologia e Serviços Ltda. 2009.

FAYOL, Henri. Administração Industrial e Geral.São Paulo. Atlas, 1990.

FLEURY, S. J. F. Redes de proteção: incentivos, escolhas e comportamentos na política brasileira. 2009. Tese (Doutorado) - Faculdade de Filosofia e Ciências Humanas da Universidade Federal de Minas Gerais, Belo Horizonte, 2009. 
HERNES, T. Four ideal-types organizational responses to the new public management reforms and some consequences. International Review of Administrative Sciences, v. 71, n. 1, p. 5-17, 2005.

HOOD, C. A public management for all seasons? Public Administration, . 69, $\mathrm{n}$. $1,1991$.

JUSTEN FILHO, Marçal. Curso de Direito Administrativo. São Paulo: Saraiva, 2005.

GOMES, Lauren Beltrão et al . As origens do pensamento sistêmico: das partes para o todo. Pensando familiar, Porto Alegre, 2014.

KOLTER, John P. Liderando Mudanças. Rio de Janeiro: Elsevier, 2013.

LAKATOS, Eva Maria; MARCONI, Mariana de Andrade. Fundamentos de Metodologia Científica. 4 ed. São Paulo: Atlas, 2001.

LIMA, Joilson Souza de. O Planejamento Estratégico como Ferramenta de Gestão. Revista Científica Multidisciplinar Núcleo do Conhecimento. Ano 03, Ed. 03, Vol. 03, pp. 58-69, Março de 2018. ISSN:2448-0959.

MEIRELLES, Hely Lopes. Direito Administrativo Brasileiro. São Paulo: Malheiros, 2002.

MANNING, N. et al. Reformas de gestão pública: o que a América Latina tem a aprender com a OCDE.In: MEDEIROS, P. C.; LEVY, E. (Orgs.). Novos caminhos da gestão pública: olhares e dilemas. Rio de Janeiro: Qualitymark; Brasília: CONSAD, 2009. p. 97-148.

MORAES, Germana de Oliveira. Controle Jurisdicional da Administração Pública. São Paulo: Dialética, 1999, p. 127.

MORGAN, Goreth. Imagens da Organização. São Paulo: Atlas, 1996. 
PIETRO, Maria Sylvia Zanella Di. Direito Administrativo. São Paulo: Atlas, 2002.

SENGE, Peter M. A quinta disciplina: arte e prática da organização que aprende. $25^{\mathrm{a}}$ ed. Rio de Janeiro: BestSeller, 2009.

SEMLER, Ricardo. Virando a própria Mesa: Uma história de Sucesso Empresarial. Rio de Janeiro, 2002.

VALENÇA, Antônio Carlos. Aprendizagem Organizacional: 123 aplicações práticas de arqueótipos sistêmicos. São Paulo:SENAC, 2011.

VASCONCELLOS, Maria José Esteves. Pensamento Sistêmico: O novo paradigma da ciência. 9aㅗ ed. Campinas-SP: Papirus,2010.

ZANELLI, José Carlos. Interação Humana e Gestão: a construção psicossocial das organizações de trabalho. São Paulo: Casa do Psicólogo, 2008.

Soumis : octobre 2019.

Approuvé : octobre 2019. 